\title{
Introduction
}

\section{Lumbar trauma}

\author{
Paul A. Anderson, M.D., ${ }^{1}$ Paul M. Arnold, M.D., ${ }^{2}$ \\ AND Robert F. HEARY, M.D. ${ }^{3}$
}

\begin{abstract}
${ }^{1}$ Department of Orthopedics and Rehabilitation, University of Wisconsin, Madison, Wisconsin; ${ }^{2}$ Department of Neurosurgery, University of Kansas Medical Center, Kansas City, Kansas; and ${ }^{3}$ Department of Neurosurgery, Rutgers New Jersey Medical School,
\end{abstract} Newark, New Jersey

This issue of Neurosurgical Focus is unique in that it represents a collaboration between the Journal of Neurosurgery Publishing Group and the Lumbar Spine Research Society. It is the first such combined effort, and the expertise of both groups will enhance our understanding of how to care for patients who sustain trauma to the lumbar spine. In the past several years, there has been an increased understanding of the management of lumbar spine disorders, including trauma. These advances have included new information in the areas of biomechanics, biologics, neuromonitoring, navigation, and surgical technique. Advances in the surgical arena are both technique related and device related. Minimally invasive surgical techniques have become popular in the last few years, as has the use of expandable cages that can allow ventral reconstruction from a posterior approach. Intraoperative spinal imaging and navigation have increased the accuracy of pedicle screw placement, and the direct lateral approach to the spine has allowed for the use of large interbody grafts to enhance fusion success.

All of this new knowledge has been translated to the management of lumbar and thoracolumbar trauma. Advances in the medical management of patients with spinal trauma have allowed injured patients to have near-normal lifespans postinjury,andthemostrecentsurgicaltechniques have also kept pace. They allow for less blood loss, earlier mobilization, shorter hospital stays, higher fusion rates, and, importantly in the current environment, lower cost.
Treatment of these injuries can be complex, and the potential for complications is high. Many patients with lumbar trauma also have abdominal, pelvic, or long-bone injuries, which may be non-weight bearing. Surgical intervention carries risks such as exaggerated blood loss, infection, neurological injury, pseudarthrosis, thromboembolic sequelae, and instrumentation failure. Issues regarding timing and the optimal surgical approach remain controversial.

The current issue of Neurosurgical Focus provides the reader with a broad overview of the latest surgical options for treating patients with lumbar spine trauma, including circumferential fusion, minimally invasive approaches, and the cement augmentation of fractures. There are also systematic reviews on the topics of surgical complications, and articles on nonsurgical management. Articles also address the timing of surgery for patients with lumbar lesions, as well as sacral fractures, and a review of pediatric lumbar trauma.

The comprehensive assessment of lumbar spine trauma, presented by spine surgeons from both orthopedic and neurological fields, is a great example of how in working together we are able to achieve lofty goals. A sincere thanks to all the authors who have contributed to this effort and, in particular, to the members of the Lumbar Spine Research Society for initiating this project. (http://thejns.org/doi/abs/10.3171/2014.5.FOCUS14196)

\section{Disclosure}

Dr. Anderson reports being a consultant for Stryker and Aesculap, owning stock in Titan Surgical and SI Bone, having ownership of Spartec and Expanding Orthopedics, and receiving royalties from Stryker and Pioneer Surgical. Dr. Arnold reports being a consultant for Medtronic Sofamor Danek, LifeSpine, Integra Life, SpineWave, Stryker Spine, FzioMed, MIEMS, AOSpine North America, and Z-Plasty; he is on the board of directors of LSRS and is a member of the NASS Professional Compliance Panel and the NASS Ethics Committee. 